\title{
Ausgewählte Probleme der Flexion der Wortartkategorie Verb in der DaF-Lehrerausbildung
}

\author{
Angrit Janakiev
}

Verbal inflection must be perceived as grammatical linguistic material that is used since the beginning of controlled L2 teaching and learning processes. Not only theoretically but also in terms of its teaching in foreign language classes, the term of verbal inflexion causes a wide range of complex matters. This paper expounds the problems of the terms flexion and finitivity (Flexion und Finitheit) as essential components of language in teaching and textbooks.

German as a Foreign Language - morphology - morphology of flexion - flexion - finitivity conjugation - verb - grammatical linguistic material

Verbflexion ist ein grammatischer Sprachstoff, der in gesteuerten L2- Lehr- und Lernprozessen von Beginn an präsent (ist) und nicht nur in theoretischer Hinsicht, sondern auch bei seiner Didaktisierung unterschiedliche Fragen aufwirft.

In diesem Beitrag sollen u. a. die Begriffe Flexion und Finitheit als wichtige Bestandteile der Unterrichts- und Lehrwerksprache problematisiert werden.

Deutsch als Fremdsprache - Morphologie - Flexionsmorphologie - Flexion - Finitheit Konjugation - Verb - grammatischer Sprachstoff

Zwar thematisiert dieser Beitrag die Verbflexion, doch ist es nicht mein Anliegen, die in einschlägigen Grammatiken nachzulesenden Beschreibungen zu repetieren. Auch beabsichtige ich keineswegs, Vorschläge zu unterbreiten hinsichtlich der Didaktisierung dieses grammatischen Sprachstoffes im Fremdsprachenunterricht.

Mein Vorhaben bezieht sich vielmehr auf einen Bereich zwischen den von Experten vorgenommenen Beschreibungen eines sprachwissenschaftlichen Gegenstandes und dessen Umsetzung in Lehr- und Lernkontexten, der den Charakter einer noch ungenügend erforschten Grauzone darstellt und der m. E. als Prozess der „Suche nach geeigneten Transfermöglichkeiten linguistischer Theorie" bezeichnet werden kann.

Zunächst muss Folgendes beachtet werden: Eine didaktisierte Fremdsprachengrammatik verlangt von den künftig Lehrenden, dass sie

- den Umfang des zu vermittelnden Sprachstoffes (so auch den der Verbflexion) sowie die Intensität, mit der dies geschehen soll, bestimmen können,

- dass sie Entscheidungen zur Gewichtung bestimmter Teilinhalte treffen können, weil in gesteuerten Lehr- und Lernprozessen nicht auf Gänze gesetzt werden kann,

- Entscheidungen hinsichtlich der Progression des gesamten Sprachstoffes fällen können, 
- Möglichkeiten erkennen, den Sprachstoffbzw. Teile dessen im Zusammenhang mit geeigneten Sachstoffen lehrend anzubieten,

- die Vielfalt von Übungssequenzen kennen und berücksichtigen sowie deren Effektivität beurteilen können,

- eine Beschreibungssprache wählen, die möglichst eindeutig und verständlich ist und

- bewusstmachende Lehrverfahren im Interesse der Entwicklung von Fremdsprachenkompetenz einsetzen.

Alle mit der Didaktisierung zusammenhängenden Überlegungen setzen im Vorfeld die Aneignung linguistischer Grundkenntnisse voraus, die im Rahmen von Lehrveranstaltungen sowie innerhalb des Selbststudiums erworben werden sollten. An dieser Stelle plädiere ich für ein gewisses Verständnis dafür, dass sowohl der Umfang der fachwissenschaftlichen Beiträge als auch die widersprüchlichen Positionen, die eben z. B. auch im Zusammenhang mit dem Gegenstand der Verbflexion in der Fachliteratur vertreten werden, insbesondere Lehramtsstudierende irritieren, da sie es sind, die im Interesse des späteren Unterrichtens nach „theoretischer Sicherheit und Eindeutigkeit" suchen.

Dieses Interesse ist durchaus nachvollziehbar, wenn man sich aus der Perspektive einer künftigen Lehrperson Folgendes bewusst macht:

Neben seiner herausragenden Rolle, einen Satz in Umfang und Ausstattung gestalten zu dürfen, scheint sich das Verb zusätzlich dadurch auszuzeichnen, dass es eine Formenvielfalt an den Tag legt, die ihresgleichen sucht. In der Regel kann ein Verb in 149 unterschiedlichen Gewändern auftauchen. Jedes Substantiv, jedes Adjektiv verhält sich in dieser Hinsicht weitaus bescheidener.

\begin{tabular}{|llll|}
\hline & 3 Personen & 2 Numeri & $=6$ \\
$\mathrm{x}$ & 6 Tempora & & $=36$ \\
$\mathrm{x}$ & 2 Konjunktive & & $=72$ \\
$\mathrm{x}$ & 2 Genera & & $=144$ \\
+ & 1 Imperativ Singular + & 1 Imperativ Plural & $=146$ \\
\hline
\end{tabular}

Die nicht zu unterschätzende Schwierigkeit besteht nun darin, dass die Formen in der Verbindung mit ihren möglichen Funktionen gelehrt und gelernt werden müssen.

Im Prozess der Satzbildung wird das (Wörterbuch-)Verb zum Textverb. Manche Verben erscheinen nach herkömmlicher Ansicht als finites, andere als infinites Verb. Manche Textverben bleiben einteilig, andere werden mehrteilig. Einteilige sind konjugiert, mehrteilige verfügen auf jeden Fall über ein konjugiertes Element. Darüber hinaus beanspruchen einteilige wie mehrteilige Textverben bestimmte Plätze im Satz. Ein Lerner, der so denkt, denkt als Textproduzent. 
Textrezipienten stehen, was die Entschlüsselung von Verbformen anlangt, vor einer Aufgabe, die sie in ähnlicher Weise auch dann zu leisten haben, wenn phraseologische Bedeutungen zu dekodieren sind: Neben den einteiligen müssen auch die mehrteiligen Verbformen, welche an unterschiedlichen Plätzen im Satz zu finden sind, finit oder infinit sind, und ggf. auch die nichtverbalen Satzelemente, die nur gemeinsam mit einem finiten Verb eine syntaktische Einheit bilden, als funktional zusammengehörend, eben als Elemente verbaler bzw. nominaler Prädikate ${ }^{1}$ erkannt und verstanden werden.

Diese beiden wesentlichen Teilfertigkeiten fremdsprachlicher Kommunikation, regelhaft entsprechende Formen der Wortartkategorie Verb bilden zu können, aber auch von der aktuellen Verbform auf deren Informationsgehalt schließen zu können, sind für den Textproduzenten wie für den Textrezipienten untrennbar mit der Kenntnissyntaktischerund semantischer Regeln derzielsprachlichen Kommunikation verbunden. Mit anderen Worten: Im Rahmen der Fremdsprachendidaktik kann hier nicht die einseitige, wenngleich aus der Perspektive der fachwissenschaftlichen Forschung legitime Fokussierung ausgewählter Aspekte eines komplexen Gegenstandes favorisiert werden. Es müssen alle drei Ebenen gleichermaßen Berücksichtigung finden. Im Interesse effektiven Lehrens und Lernens besteht die Kunst der Lehrperson darin, alle Ebenen gleichzeitig im Blick zu haben.

Motivierte DaF-Lehramtsstudierende werden sich spätestens nach ihrer sprachwissenschaftlichen Ausbildung die Frage stellen, wie sie ihr erworbenes linguistisches Fachwissen zur Verbflexion in den Lehrprozess einbringen sollen, damit die Lerner im Zusammenhang mit der Produktion und Rezeption deutschsprachiger Texte zunächst bewusst darauf zurückgreifen können. Als Ziel fremdsprachlichen Lernens wird die automatisierte Beherrschung der Regeln angestrebt, welcher der schwierige Prozess des Bewusstmachens bzw. des Bewusstwerdens vorausgeht (vgl. RAMPILLON: 1996, 51; ECKERTH/RIEMER: 2000, 228).

Unter Einbeziehung dieser lerntheoretischen Sicht wird die Frage relevant, welche aus der Fachliteratur bekannten Konzepte und welche Beschreibungssprache sich im Hinblick auf die spätere berufliche Tätigkeit als praktikabel bzw. nur in modifizierter Form als umsetzbar erweisen könnten. Es erfolgt die bereits erwähnte Suche nach geeigneten Transfermöglichkeiten der linguistischen Theorie in eine „adaptierte Theorie“ für unterrichtspraktische Zwecke, die u. a. ihren Ausdruck in einer geeigneten Unterrichts- und Lehrwerksprache findet.

1 ,Prädikat' wird hier im Sinne der traditionellen Grammatik in einem engen Sinn als die Satzkonstituente verstanden, die eine auf das Subjekt bezogene Handlung mit Hilfe eines Verbs oder eines Verbalkomplexes bezeichnet. Prädikate verfügen über einteilige oder mehrteilige Strukturen. Letztere wiederum können neben ausschließlich verbalen Anteilen auch nominale Elemente enthalten. Solche mit Kopula- oder Funktionsverben verknüpften Nominal-, Adjektiv-, Pronominaloder Präpositionalphrasen werden als mehrteilige nominale Prädikate angesehen. 
Notwendiger Bestandteil dieser Beschreibungssprache sind Fachtermini. Sie übernehmen eine Art Mittlerfunktion in allen didaktischen Teilphasen gesteuerter L2-Erwerbsprozesse, weil mit ihrer Hilfe Interaktionen ausgelöst oder steuernd begleitetwerden.Im Hinblickaufdienoch unzureichend erforschteGrauzonezwischen den von Experten vorgenommenen Beschreibungen eines sprachwissenschaftlichen Gegenstandes und dessen Umsetzung in Lehr- und Lernkontexten muss die Frage beantwortet werden, ob und wenn ja, welche terminologischen Festlegungen für die Lehrsituation praktikabel sind.

So zählen ,Flexion' und ,Finitheit' u. a. zu den Termini, die in das Umfeld des Gegenstandes Verbflexion gehören und auf die während der Arbeit an diesem grammatischen Sprachstoff nur schwer zu verzichten ist. In der linguistischen Fachliteratur wird deren Begrifflichkeit recht unterschiedlich diskutiert.

Im Verlauf meines Beitrages verfolge ich das Ziel, zu begründen, welche der zu diesen beiden Beispielen in der fachwissenschaftlichen Diskussion vorliegenden Ergebnisse aufgegriffen werden sollten.

Wichtiger Bezugspunkt des Nachdenkens darüber, wie sich linguistische Auffassungen einerseits und didaktische Notwendigkeiten andererseits miteinander vereinen ließen, kann neben einschlägigen Grammatiken u. a. die von Radtke bereits 1998 erschienene Monographie „Kategorien des deutschen Verbs“ (RADTKE: 1998) sein. Dabei will ich nur zwei terminologische Festlegungen, die Radtke als Synthese der Auseinandersetzung mit ausgewählten theoretischen Konzepten (BERGENHOLTZ/MUGDAN: 1979; EISENBERG: 1994; WURZEL: 1984) formuliert, aufgreifen und in den Kontext von Lehr- und Lernprozessen der L2 Deutsch stellen.

\section{1. „Erstens: Sämtliche Verbformen, die sich aus einem Basismorphem und einem grammatischen Marker zusammensetzen, bezeichne ich als flektiert.“ (RADTKE: 1998, 42)}

Der Inhalt dieses Zitates verweist auf das Verhältnis, in welchem Verbform und Verbflexion stehen, indem die Bedingung genannt wird, die genau erfüllt sein muss, damit eine Verbform als eine flektierte eingestuft werden kann. Diese Bedingung betrifft die Verfahren, nach denen Verbformen gebildet werden. Dieser Aspekt ist für den Fremdsprachenunterricht auf jeden Fall bedeutsam.

Wenn Radtke die Ansicht vertritt, nur bestimmte Verbformen sollten als flektierte bezeichnet werden, gleichzeitig jedoch dafür plädiert, Flexion vom Begriffspaar ,finit/infinit' zu trennen (vgl. Abschnitt 3), so kann es sich bei der Menge der nichtflektierten Verben, das impliziert der Inhalt des Zitates, nur um die Formen handeln, bei denen stattgefundene Derivation zu einer Modifizierung der lexikalischen Bedeutung eines einfachen Verbs geführt hat (sprechen $\rightarrow$ besprechen/ versprechen usw.). Verbformbildungen, so lässt sich schlussfolgern, führen je nach Verfahrenstyp zu zwei unterschiedlichen Subklassen, 
a) zu verbalen Wortbildungskonstruktionen sowie

b) zu flektierten Verben

und meinen damit zwei verschiedene Perspektiven, durch die das Lehren und Lernen von Verbformen motiviert ist. Da erstere nicht Gegenstand der Flexionsmorphologie ist, wird sie an dieser Stelle nicht weiter beachtet.

Der Begriff ,Flexion' (lat. flexum, Gebogenes') ist in jenen Sprachen, in denen Lexikonwörter je nach ihrer in der mündlichen oder schriftlichen Rede eingenommenen syntaktischen Funktion in abgewandelter, geformter Weise auftreten, das ausschlaggebende Charakteristikum dafür, die betreffende Sprache (hier: die deutsche Sprache) dem flektierenden Sprachtyp zuzuordnen. Folgt man der Schulgrammatik, so verfügen die prototypischen Vertreter von sechs der zehn postulierten Wortartkategorien über die Eigenschaft der Flexion, die sich in den Flexionsarten Konjugation (Verb), Deklination (Substantiv, Artikel, Pronomen, Adjektive und Numeralien) und Komparation (Adjektiv) ausdrückt. Die Flexionsart ist einer der Klassifikatoren, nach denen Wortmaterial kategorisiert werden kann. Jeder der drei Flexionsarten sind bestimmte Flexionskategorien zugewiesen. Für das Verb handelt es sich nach traditioneller Auffassung um die Kategorisierungen Person, Numerus, Tempus, Modus und Diathese i. e. S. ${ }^{1}$ Der Begriff, Verbflexion ${ }^{\prime}$ assoziiert mit dem Prozess der Variation der formalen Seite des Verbs die damit gleichzeitig verbundene Spezifizierung der Verbbedeutung. Nicht nur Radtke will im Interesse einer sauberen Argumentation eine derartige Vermischung der unterschiedlichen Ebenen der Sprachbetrachtung vermeiden und demzufolge die Fragen der Wortformbildung getrennt von funktional-semantischen Aspekten wissen.

Diesem Gedanken möchte ich folgen und zunächst ausschließlich die Bildung flektierter verbaler Wortformen im Blick behalten mit dem Ziel zu prüfen, ob nachstehende Erklärungsansätze auch für gesteuerte L2-Lehr- und Lernprozesse umsetzbar sein könnten.

In Anlehnung an die lateinische Grammatik werden ,Verbflexion' und ,Konjugation' synonym verwendet, meinen ursprünglich jedoch nur die formale Verbindung eines Verbstamms mit einer Endung: „Von Flexion spricht man allgemein dann, wenn die betrachteten sprachlichen Einheiten Wortformen in einem strikten Sinn sind, also synthetische Formen [...] Ein Verb konjugieren (,verbinden') meint, seinen Stamm mit den Endungen zusammenzurücken" (EISENBERG: 1994, $144 \mathrm{f}$.$) .$

Radtke vertritt die Meinung, dass dieser traditionelle Konjugationsbegriff für die Beschreibung der deutschen Grammatik ungeeignet sei. Für Studium und Lehre kann m. E. jedoch festgehalten werden, dass der Terminus ,Konjugation“

1 Gelegentlich wird der Aspekt als sechste Kategorie genannt (vgl. HENTSCHEL/VOGEL: 2009, 445). 
längst nicht mehr in der Enge verstanden wird, wie sie in Bezug auf die lateinische Grammatik gilt, sondern schon seit langem eine Begriffserweiterung erfahren hat. Auch die Schulgrammatik ist ein Beleg dafür, dass sich die Beschreibung der Verbflexion keineswegs darauf beschränkt, was der Terminus im althergebrachten Sinne meint. Beispielsweise gelten die Verbformen las und schrieb als konjugierte Formen von lesen bzw. schreiben, ohne dass ein verbindendes Prinzip für die Formenbildung erkennbar wäre, und Verbformen wie gelesen oder geschrieben, die strukturell betrachtet, die entsprechende Bedingung genau erfüllen, gelten hingegen als nicht konjugiert. Bei der Verbform gäbe, die eindeutig zu den konjugierten zählt, wird deutlich, dass außer der Addition von Stamm und Endung ein zusätzliches Verfahren registriert werden kann, das zur Bildung der Verbform geführt hat: die Abwandlung des Stammes. Auch hierbei dürfte es sich nicht um Konjugation im strengen Sinne handeln. Des weiteren treffen wir im Deutschen auf die besonders hoch frequenten mehrteiligen (d. s. analytische oder auch: periphrastische) Verbformen (hat geschrieben, ist gelaufen), die aus dem, was der traditionelle Begriff Konjugation meint, gleichermaßen herausfallen (vgl. RADTKE: 1994, 13f.; LEISS: 1992, 41; EISENBERG: 1998, 144).

Radtke schließt sich im Ergebnis der Auseinandersetzung mit anerkannten Konzepten den Überlegungen Wurzels (WURZEL: 1984) an, der für eine weite Auffassung des Begriffes, Verbparadigma' plädiert: Würde man ,Flexion` nicht i. e. S. (als einen Prozess, in dessen Folge die Verbindung eines Verbalstammes mit einem Flexionsmorphem zu einem finiten Verb führt) definieren, so könne das Verbparadigma allen flektierten Verbformen Platz bieten.

Im Hinblick auf mein eingangs formuliertes Ziel werde ich auf dieses Konzept von Wurzel Bezug nehmen und aus der Sicht eines Lerners der L2 Deutsch in aller Kürze darstellen, welche unterschiedlichen formalen Verfahren die Entstehung einer flektierten verbalen Wortform bewirken:

Für den Lerner in seiner Sprecher-/Schreiberfunktion geht es um das Kennen und Nutzen der Verfahren, die im Zusammenhang mit der Enkodierung zielsprachlicher Verbbedeutungen aktiviert werden müssen. Die Formenbildung deutscher Verben erfordert neben der Kenntnis auch die mehr oder weniger automatisierte Anwendung dieses Regelwissens. Für die Verbflexion müssen morphologische und syntaktische Regeln beherrscht werden. Eine Regel zu beherrschen, äußert sich in der Fähigkeit, eine Verbform bilden zu können, indem man mit den für die betreffende Sprache vorhandenen Mitteln erwartungsgemäß operiert. Typische Operationen, die für die Verbflexion im Deutschen gelten, sind die Kombination (Addition), Modifikation und Subtraktion. Während das verbale Basismorphem in jedem Fall das „Ausgangsmaterial“ ist, auf dessen Grundlage die flektierte Verbform entsteht, lässt sich der grammatische Marker als das „Angebot an Werkzeugen“ begreifen. Mit diesen muss auf der Wort- oder aber auf der Satzebene regelhaft operiert werden. Auf der Wortebene zählen nach herkömmlicher Redeweise die 
grammatischen Suffixe, der Ablaut, Zirkumfixe und autonom auftretende verbale Stammmorphe bzw. Stamm-Allomorphe zu den sogenannten morphologischen Markern. Grammatische Marker auf der Satzebene bezeichnet Wurzel als syntaktische Marker: „Syntaktische Marker sind [...] solche Marker, die grammatische Kategorien nicht am Wort, sondern durch die Wortfolge [...] repräsentieren" (WURZEL: 1984, 60). Nachstehende Anmerkung möchte ich ergänzen: So, wie flektierte verbale Wortformen auch im Ergebnis des Zusammenwirkens unterschiedlicher morphologischer Marker (bspw. geschrieben: Kombination und Modifikation) entstehen können, ist das Operieren mit syntaktischen Markern untrennbar an das Operieren mit morphologischen Markern gebunden.

\section{Grammatische Marker}

zur Bildung flektierter verbaler Wortformen (vgl. WURZEL: 1984, 60 f. und RADTKE: 1998, 35 ff.)

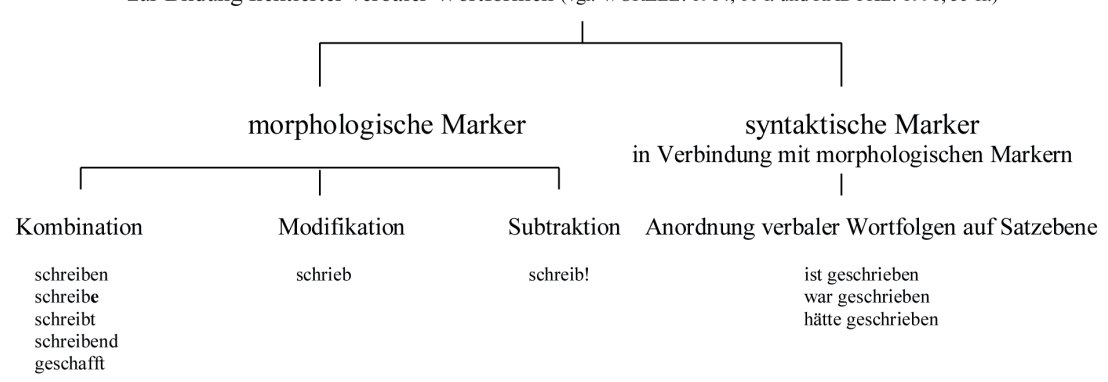

Bezieht man die Theorien zum gesteuerten Zweitspracherwerb in diese Überlegungen ein, scheint die Konzentration auf den operationalen Aspekt, unter dem die Formenbildung der Vertreter flektierbarer Wortartkategorien beschreibbar ist, sehr geeignet, wenn vielleicht auch ungewöhnlich, weil auch gegenwärtig dem mechanischen (Auswendig-)Lernen der Formenvielfalt weiterhin große Aufmerksamkeit geschenkt wird, obwohl bereits bekannt ist, dass bewusstmachende Lehrverfahren das Lernen positiv steuern helfen. Lerner interessieren sich m. E. zunächst für das WIE, bevor sie das WAS beherrschen. Verfahren transparent zu machen, zu demonstrieren, auf welche Art und Weise zielsprachliche Wortformen gebildet werden und in welcher Art und Weise man komplexe Verbformen verstehen lernen kann, entspräche dem besonderen Anliegen des Lehrens und Lernens einer L2. In Anbetracht der Tatsache, dass der Prozess des Fremdsprachenlernens vor dem Hintergrund der eigenen Muttersprache und ggf. auch vor dem weiterer bereits gelernter Sprachen erfolgt, könnte ein operationaler Ansatz zudem vor allem für erwachsene Lerner effektiv sein.

Würde im praktischen Fremdsprachenunterricht ,Flexion' in dieser Weite definiert werden, wie Radtke es nahe legt, sind ,Flexion' (hier: ,Verbflexion') und 
„Konjugation' terminologisch nicht mehr austauschbar, denn es werden auch solche Verbformen erfasst, die mit dem Begriff, den wir traditionell von ,Konjugation“ haben, unvereinbar sind: Einem Verb ging Konjugation voraus, wenn ihre synthetische bzw. analytische Form Ausdruck der in ihr enkodierten grammatischen Kategorien (oder besser: Verbalkategorien) ist: „Verben werden konjugiert: Sie flektieren (verändern ihre Form) im Hinblick auf die Kategorienklassen Tempus, Modus, Numerus und Person [sowie Diathese, sofern die lexikalische Verbbedeutung die Bildung eines Passivs zulässt, A. J.]“ (DUDEN: 2005, 395).

Wenn nun Konjugation neben der formalen auch den funktional-semantischen Aspekt eines Verbs einschließt, Form- und Bedeutungsseite nach Radtke terminologisch jedoch konsequent voneinander unterschieden werden sollen, so kann nach Wurzel und Radtke auf den im Sinne der Schulgrammatik verstandenen Begriff ,Konjugation' verzichtet werden. Der weite Begriff von ,Flexion' ist Gegenstand der Grammatik (der Flexionsmorphologie), wie Wurzel und Radtke es favorisieren. Dieser weite Begriff von ,Flexion' fokussiert ausschließlich die formale Seite des Textverbs, zielt auf Struktur und Bildung deutscher Textverben, beschreibt Prozesse und Verfahren, welche verbale Strukturen zur Folge haben.

Gegenstand der Wortbildungsmorphologie hingegen sind Wortbildungskonstruktionen, die sich, wenn man sie ebenfalls auf ihren ausschließlich formalen Aspekt reduzieren will, von flektierten Verbvorkommen darin unterscheiden, dass ihrer Bildung ein Verfahren zugrunde liegt, das keine grammatischen Marker, sondern Wortbildungsaffixe nutzt.

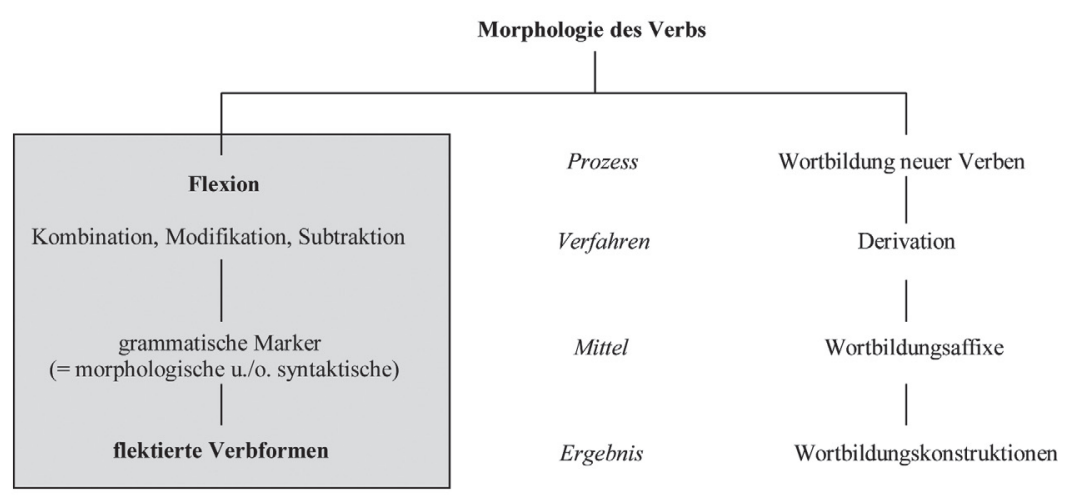

2. „Zweitens: Die Begriffe,Flexion' und ,Finitheit' werden voneinander getrennt; flektierte Verben sind nicht notwendigerweise finit. Finit sind Verbformen genau dann, wenn sie dazu dienen, das Bündel der Verbalkategorien auszudrücken [...]“ (RADTKE: 1998, 42) 
Mit ihrer zweiten terminologischen Festlegung will Radtke klären, dass ,Flexion“ und ,Finitheit ${ }^{`}$ zwei unterschiedliche Ebenen der Diskussion betreffen, die nicht miteinander vermischt werden sollten. Erst wenn bestimmte Voraussetzungen erfüllt sind, kann einer bestimmten Menge flektierter Verbformen das Merkmal Finitheit zugesprochen werden, was wiederum eine Unterscheidung von denjenigen flektierten Verbformen ermöglicht, denen dieses Merkmal fehlt. Finitheit wird als Folge enkodierter Verbalkategorien angenommen oder anders ausgedrückt: Nur denjenigen flektierten Verbformen ist das Merkmal Finitheit zuzuordnen, denen der Status zukommt, formaler Ausdruck enkodierter Verbalkategorien zu sein.

Auf den ersten Blick wird deutlich, dass die Klärung eines jeden Terminus' neue Fragen aufwirft. Will man ,Finitheit' im Kontext der, Verbalkategorien' diskutieren, stößt man auch bei diesem Terminus in der Fachliteratur verständlicherweise auf begriffliche Unterschiede, so dass die Unterrichtspraxis nach einer Positionierung verlangt. Machen wir uns Folgendes bewusst:

Verbformen vermitteln neben ihrer konkreten lexikalischen Bedeutung grammatische Informationen, die mit ihrer kategoriellen Wortartbedeutung in Verbindung stehen. ${ }^{1}$

Im Interesse einer didaktisierten Grammatik sollte die Beschreibung der einzelnen Kategorisierungen, die auf repräsentationistischen oder instrumentalistischen Erklärungsansätzen (vgl. RADTKE: 1998, 44) beruhen, m. E. in vereinfachender Weise vorgenommen werden. Während Finitheit die formale Gemeinsamkeit der Verbalkategorien ist, unterscheiden sich diese in funktional-semantischer Hinsicht: Die Kategorien desTempus, Modus und der Diathesespezifizieren das Verbalgeschehen in Hinblick auf Zeitlichkeit, Geltungsgrad und Perspektivität. In den Kategorien der Person und des Numerus äußern sich dagegen die Rollenverteilung im Satz (sprechende/angesprochene Person/besprochene Person bzw. Besprochenes) und gleichzeitig die Anzahl der jeweiligen Personen bzw. Dinge in ihrer entsprechenden Rollensituation. Die formale Markierung der beiden letztgenannten grammatischen Kategorien kann aus strukturell-syntaktischer Sicht als der explizite Ausdruck für die inhaltliche Beziehung zwischen dem grammatischen Subjekt und dem Prädikat betrachtet werden. $\mathrm{Ob}$ es angemessen wäre, diesen beiden Kategorien satzinterne Funktionen und den drei erstgenannten eher satzexterne Funktionen zuzusprechen, soll an dieser Stelle nicht geklärt werden.

Der Terminus ,Verbalkategorien' wird vor allem hinsichtlich seiner Enge bzw. Weite diskutiert. Das äußert sich in der Frage, ob diese in ihrer Gänze oder nur zu einem Teil in der Verbform verschlüsselt sein müssen, damit die Bedingungen zutreffen, die für Finitheit gelten sollen.

1 Auf eine Charakterisierung der unterschiedlichen Modelle, die sich in der Fachliteratur zu den Verbkategorisierungen finden lassen, und zu ihrem Beitrag, den diese für die Satzbedeutung leisten, verzichte ich an dieser Stelle bewusst. 
Hinsichtlich der Voraussetzungen, unter denen Verbvorkommen als finit bezeichnet werden, sind es zum einen solche Auffassungen, nach denen bereits bei der Verschlüsselung einer Verbalkategorie von Finitheit gesprochen wird (GLÜCK: 2000, 210). Eine andere Version legt zugrunde, es handele sich um die nach Person und Numerus flektierende Form ${ }^{1}$ (BLATZ: 1896, 441; SCHANEN: 1995, 52).

Radtke und Eisenberg hingegen vertreten wie Bußmann die Position, eine Verbform nur dann als finit zu identifizieren, wenn diese jeweils eine Information aus dem gesamten Bündel der Verbalkategorien (Person, Numerus, Tempus, Modus, Genus verbi) auszudrücken imstande ist (du schreibst. 2. Person, Singular, Präsens, Indikativ, Aktiv) (vgl. BUSSMANN: 1990, 243; RADTKE: 1998, 42; EISENBERG: 1994, 177; aber auch GLÜCK: 2000, 209). Damit wird ein weiter Begriff von ,Finitheit' favorisiert. Allerdings nutzen Eisenberg und Radtke ,Finitheit ${ }^{\star}$ in zweierlei Hinsicht: „Wir folgen hier einem weit verbreiteten Sprachgebrauch und nennen auch periphrastische Formen finit, die eine einfache finite Form als Bestandteil enthalten" (EISENBERG: 1994, 191). Radtke formuliert dies explizit als ihre vierte terminologische Festlegung im Zusammenhang mit der Beschreibung des morphologischen Status der Verbalkategorien: „Da die Verbalkategorien im Deutschen nicht nur auf morphologischem, sondern auch auf syntaktischem Wege symbolisiert werden, muss man auch zusammengesetzte Formen berücksichtigen. Eine Form wie wird geschrieben drückt die Kategorie Passiv genau durch das Zusammenspiel von morphologischen und syntaktischen Markern aus. Aus eben diesem Grund gilt für mich die gesamte Form als finit und nicht nur das Hilfsverb, das üblicherweise als der finite Bestandteil der Konstruktion angesehen wird“ (RADTKE: 1998, 41).

Wenn ich die Funktion der flektierten Verbform fokussieren will und es dabei als unerheblich ansehe, ob die Verbalkategorien auf synthetischem oder analytischem Weg ausgedrückt sind, muss ich diese Position gerade auch im Hinblick auf unterrichtspraktische Zwecke zunächst stützen.

Analog zum Begriff, ,Finitheit' tragen dann diejenigen Verbvorkommen das Merkmal Infinitheit, bei denen es sich nicht um den formalen Ausdruck jeweils einer Information aus dem verbalen Kategorienbündel handelt bzw. bei denen nur ein Teil aus dem Kategorienbündel abgebildet ist. Dazu rechnen der Infinitiv sowie die Partizipien I und II. Auch die beiden Imperativformen, für die keine explizit ausgedrückte Personenmarkierung vorliegt, erfüllen demzufolge die Bedingung für Finitheit nicht, sie sind bspw. bei Eisenberg und Radtke ebenfalls den infiniten Formen zugeordnet und verfügen damit auch nicht über den Status einer Subkategorie der Verbkategorisierung Modus. Infinite flektierte Verbalformen gelten damit zwar als Kategorien der Wortart Verb, erfüllen aber nicht die Bedingung, die diesem Konzept zufolge für Verbalkategorien festgelegt ist.

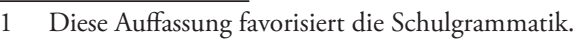


Die wesentlichsten Gedanken möchte ich noch einmal zusammenfassen: Nach Eisenberg und Radtke ist Finitheit Ausdruck einer formalen Gemeinsamkeit flektierter Verben. Finit ist eine flektierte Verbform nur dann, wenn sie jeweils eine Kategorie aus dem gesamten Kategorienbündel aufweist. Die Eigenschaft Finitheit ist nicht an synthetisch realisierte Verbalkategorien gebunden, sondern gilt auch dann, wenn diese auf analytischem Weg umgesetzt sind. Wenn, wie anfangs gesagt, ein prototypisches Verb 149 unterschiedliche Formen aufweisen kann, so gelten nach diesem Konzept alle 149 als flektiert, von diesen 144 als finit und fünf als infinit.

An dieser Stelle behaupte ich, dass ein weiter Begriff von, Finitheit' in gesteuerten Lehr- und Lernprozessen wenig praktikabel ist. Folgende Argumente stützen diese These:

1. Aus der Fremdsprachenperspektive kann ein gelegentlicher Blick in die Wortgeschichte hilfreich sein: ,finit' bezeichnet zunächst eine Art der Begrenzung, Bestimmung der betreffenden flektierten Verbform. Wer oder was begrenzt wen oder was und in welcher Hinsicht? Wodurch kann eine solche Verbform begrenzt, bestimmt sein? Inwiefern kann eine solche Verbform selbst Grenzen setzen? Wem setzt sie eine Grenze? Die Grenzen werden und sind zugleich gesetzt aufgrund ihrer mit morphologischen Mitteln ausgedrückten aktuellen Form.

2. Für das Deutsche ist die formalgrammatische Kongruenz in den Kategorisierungen Person und Numerus, in denen das Verb mit seinem grammatischen Subjekt übereinstimmen muss, obligatorisch und expliziter Ausdruck der syntaktischen Zusammengehörigkeit beider Konstituenten. Im Deutschen wird die inhaltliche Beziehung zwischen dem Verbalgeschehen und dem Handlungsträger - das gilt für prototypische Satzkonstruktionen - auf der syntaktischen Ebene als Interdependenzbeziehung mit Hilfe eines morphologischen Markers genau an einer Position signalisiert: Die synthetische Verbform selbst bzw. ein Element eines mehrteiligen verbalen bzw. ein verbales Element eines nominalen Prädikats kongruiert mit dem grammatischen Subjekt in Person und Numerus. (Du schreibst die Mail./ Er hat die Mail geschrieben./ Sie legen Wert auf das Schreiben einer Mail?! Ich habe auf das Schreiben einer Mail Wert gelegt). Die aktuellen Personen- und Numeruskategorien des grammatischen Subjekts begrenzen genau einen Teil der Verbalphase hinsichtlich deren aktueller Form.

Anders ausgedrückt: „Das eigentliche, d. h. finite Verb hat im Deutschen immer mindestens eine ,Leerstelle' der obligatorischen Fügungspotenz, namentlich die der Fügung mit dem grammatischen Subjekt [...]“ (ADMONI: 1966, 161).

Dieser Aspekt ist insbesondere für den L2-Textproduzenten relevant. Verstöße gegen die Norm, die Interdependenzbeziehung formal exakt zu kennzeichnen, sind in gesteuerten und ungesteuerten Sprachproduktionsprozessen der Zielsprache 
Deutsch ein eindeutiges Indiz für einen noch recht geringen Lernstand. Das Satzverb bzw. das Verb mit Spezialfunktion wird im frühen Lernstadium unmarkiert gesetzt.

3. Sprecherintentionen realisieren sich auch über die grammatische Kategorie der Satzart. ${ }^{1}$ Satzarten erfordern, ohne dass Erscheinungen der Polysemie und Synonymie $^{2}$ ausgeschlossen werden, eine besondere Positionierung der verbalen Konstituente mit Person- und Numerusmarkierung und gelten insofern gleichzeitig auch als ein wichtiger „Wegweiser“ des deutschen Satzbaus.

4. Ein synthetisch ausgedrücktes Verbalgeschehen ist neben den Kategorien Person und Numerus, über die es syntaktisch direkt mit dem Handlungsträger verbunden ist, gleichzeitig auch in Bezug auf Tempus, Modus und Genus markiert und damit Träger aller Verbalkategorien. Insofern weist eine solche flektierte Verbform einen besonderen Status gegenüber analytisch ausgedrückten Verbalkategorien auf. Würde man einer analytischen flektierten Verbform insgesamt das Merkmal Finitheit zusprechen, so überzeugt das aufgrund der Tatsache, dass alle Elemente erst in ihrer Gemeinsamkeit das Bündel der Kategorien realisieren. Innerhalb einer solchen Verbstruktur müssen aus didaktischen Gründen allerdings Konstituenten, die zur Realisierung der Verbalkategorien nur beitragen, ohne selbst die so wichtige Interdependenzbeziehung markieren zu können, terminologisch von eben diesen unterschieden werden.

5. Der L2-Lerner handelt jedoch nicht nur als Textproduzent. Geht man von der Annahme aus, zielsprachliche Textrezeption erfolge sowohl über die Fähigkeit, Satzelemente auch hinsichtlich ihrer Wortartbedeutung entschlüsseln zu können, sowie über die Fähigkeit, Einwort- und Mehrwortphrasen aktuelle syntaktische Funktionen zuweisen zu können, so wird der in 1.-4. favorisierte traditionelle Begriff von Finitheit keineswegs beiden Seiten fremdsprachlicher Kommunikation gerecht: Die im Satzprädikat enkodierten wichtigen semantischen Informationen (Zeitlichkeit, Geltungsgrad der Äußerung und Perspektivität des Geschehens) werden ausgeblendet. Für das Verstehen der "Satzaussage“ spielen demzufolge alle zum Prädikat gehörenden Elemente eine Rolle. Die in Person und Numerus mit dem Subjekt kongruierende Verbform bspw. ist erst in dem Moment finit („bestimmt“ im Sinne Eisenbergs und Radtkes), wenn sie in den Zusammenhang mit den übrigen das Prädikat konstituierenden Elementen gestellt wird.

1 Sätze können hinsichtlich ihrer Modalität nach Deklarativ-, Interrogativ-, Imperativ-, Optativund Exklamativsätzen unterschieden werden. Die Platzierung des Verbs, das über die Personenund Numerus-markierung verfügt, kann als ein mögliches formales Mittel zur Realisierung der Sprecherintention gewertet werden (vgl. DÜRSCHEID: 2000, 62).

2 Einer bestimmten Satzstruktur (Verberst-, Verbzweit-, Verbletztstellung) ist mehr als nur ein Satzmodus zugeordnet und umgekehrt lässt sich ein bestimmter Satzmodus über verschiedene Satzstrukturen syntaktisch signalisieren. 
6. Gegen einen weiten Begriff von Finitheit spricht aus der Perspektive des Textrezipienten allerdings, dass die Entschlüsselung der Satzinformationen vermutlich nicht linear, sondern "schichtweise“ erfolgt. Auf die Frage, ob für das schrittweise Satzverstehen das Erschließen des Satzkerns oder eher das Erfassen des Satzminimums ${ }^{1}$ bedeutsam ist, gibt es bislang keine Antwort. In beiden Fällen scheinen jedoch die finite Verbform i. e. S. und das damit gleichzeitig verbundene Erkennen der mit ihr in Person und Numerus kongruierenden Nominativphrase den Ausgangspunkt der Dekodierung zu bilden.

Im Interesse beider Perspektiven, der eines L2-Textproduzenten sowie der eines L2-Textrezipienten, stelle ich nach der Prüfung der diesem Beitrag zugrunde liegenden Konzepte der germanistischen Linguistik folgende Standpunkte als Fazit zur Diskussion:

a) Ein weiter Begriff von Flexion wird unterstützt, da mit dessen Hilfe auf das "grammatische Verhalten“ der Vertreter der Wortartkategorie Verb aufmerksam gemacht wird.

b) Der weite Begriff von Flexion orientiert auf die Frage nach der Formenvielfalt, nach möglichen Strukturen und Operationen einschließlich ihrer Mittel. Den Fokus auf operationale Aspekte zu richten, ist im Interesse bewusstmachender Lehrverfahren die effektive Alternative zum mechanischen Lernen.

c) Flektierte Verbformen lassen sich hinsichtlich ihrer Funktionen in zwei Subklassen gliedern: Vertreter der Gruppe 1 sind in der Lage, die lexikalische Bedeutung des Satzverbs über Verbalkategorien in Hinblick auf Zeitlichkeit, Geltungsgrad, Perspektivität und Rollenverhältnisse allein oder beitragend zu spezifizieren. Dieser Leistung entsprechen synthetische bzw. analytische Strukturen. Die syntaktische Funktion der Vertreter der Gruppe 1 liegt darin, entweder allein oder aber beitragend das Prädikat des Satzes ausdrücken zu können. Der alleinige Vertreter der Gruppe 2, das Partizip I, ist kein eigentliches Verb mehr, sondern der adjektivische (nominale) Ausdruck für dessen verbalen Inhalt. Das Partizip I kann drei syntaktische Funktionen übernehmen: Es kann als Attribut, Adverbial oder Prädikatsnomen auftreten. In jedem Fall lässt sich mittels Transformation die vom Verb vererbte prozessuale Grundbedeutung nachweisen. (Eine schleppende Verhandlung/ die Verhandlung verläuft schleppend/ die Verhandlung ist schleppend $\rightarrow$ Die Verhandlung schleppt sich dahin.)

d) Beim Umgang mit dem Begriff Finitheit plädiere ich aus fremdsprachendidaktischer Sicht für einen engen Begriff, da der

1 Satzkern wird vom Satzminimum insofern unterschieden, als der Satzkern die syntaktische Beziehung zwischen dem Subjekt und dem einteilig oder mehrteilig strukturierten verbalen bzw. nominalen Prädikates meint, während das Satzminimum alle durch die Valenz des satzgründenden Verbs geforderten obligatorischen Mitspieler umfasst. 
damit gemeinten Verbform sowohl in sprachrezeptiven wie auch in sprachproduktiven Prozessen eine besondere Funktion zukommt.

e) Synthetische Verbalstrukturen sind immer finit, analytische Verbalstrukturen weisen finite und infinite Konstituenten auf. Über das Merkmal Finitheit verfügen diejenigen flektierten Verbformen, die mithilfe eines morphologischen Markers die inhaltliche Beziehung zwischen dem grammatischen Subjekt und dem Prädikat über formalgrammatische Kongruenz in den Verbalkategorien Person und Numerus symbolisieren.

f) Verbalkategorien sind das Ergebnis der Kategorisierung flektierter Verbformen nach den Kategorisierungen Person, Numerus, Tempus, Modus und Diathese.

g) Alle flektierten Verbformen, die allein oder beitragend diese Verbalkategorien ausdrücken können, zählen zum Verbparadigma.

h) Ein im Einzelfall lückenhaftes Verbparadigma kann seine Ursache u. a. in der Verbbedeutung haben. Durchaus bildbare Formen wären sinnfrei (Es wird geregnet).

\section{Literatur:}

ADMONI (1966): Admoni, Vladimir Grigorievitsch. Der deutsche Sprachbau. Moskau, Leningrad: Leningrader Verlag, 1966.

BERGENHOLTZ/MUGDAN (1979): Bergenholtz, Henning/Mugdan, Joachim. Einführung in die Morphologie. Stuttgart, Berlin, Köln, Mainz: Kohlhammer Verlag, 1979.

BLATZ (1896): Blatz, Friedrich. Neuhochdeutsche Grammatik mit Berücksichtigung der historischen Entwicklung der deutschen Sprache. 2. Bd.: Satzlehre (Syntax). Karlsruhe: Peter Lang, 3. Aufl., 1896.

BUSSMANN (1990): Bußmann, Hadomod. Lexikon der Sprachwissenschaft. Stuttgart: Kröner Verlag, 2. Aufl., 1990.

DUDEN (2005): Duden Bd. 4. Die Grammatik der deutschen Gegenwartssprache. Hg. von der Dudenredaktion. Mannheim: Bibliographisches Institut \& F.A. Brockhaus, 2005.

DÜRSCHEID (2000): Dürscheid, Christa. Syntax. Grundlagen und Theorien. Wiesbaden: Westdeutscher Verlag, 2000.

ECKERTH/RIEMER (2000): Eckerth, Johannes; Riemer, Claudia. Awareness und Noticing als Bindeglied zwischen kognitiven und affektiven Faktoren des Fremdsprachenlernens, in: Riemer, Claudia (Hg.): Kognitive Aspekte des Lehrens und Lernens von Fremdsprachen. Tübingen: Gunter Narr Verlag, 2000.

EISENBERG (1994): Eisenberg, Peter. Grundriss der deutschen Grammatik.. Stuttgart: Metzler Verlag, 3. Aufl., 1994.

EISENBERG (1998): Eisenberg, Peter. Grundriss der deutschen Grammatik. Bd. 1: Das Wort Stuttgart: Metzler Verlag, 1998.

GLÜCK (2000): Glück, Helmut (Hg.). Metzler Lexikon Sprache. Stuttgart, Weimar: Metzler Verlag, 2. Aufl., 2000.

HENTSCHEL/VOGEL (2009) Hentschel, Elke/Vogel, Petra M. Deutsche Morphologie. Berlin, New York: Walter de Gruyter, 2009.

LEISS (1992): Leiss, Elisabeth. Die Verbalkategorien des Deutschen. Ein Beitrag zur Theorie der sprachlichen Kategorisierung. Berlin, New York: Walter de Gruyter, 1992. 
RADTKE (1994): Radtke, Elke. Die Kategorien des deutschen Verbs. Zur Semantikgrammatischer Kategorien. Tübingen: Gunter Narr, 1998.

RAMPILLON (1996): Rampillon, Ute. Lerntechniken im Fremdsprachenunterricht. Ismaning: Hueber, 3. Aufl., 1996.

SCHANEN (1995): Schanen, Francois. Grammatik Deutsch als Fremdsprache. München: iudicium Verlag, 1995.

WURZEL (1984): Wurzel, Wolfgang U. Flexionsmorphologie und Natürlichkeit. Ein Beitrag zur morphologischen Theoriebildung. Berlin: Akademie Verlag, 1984. 